
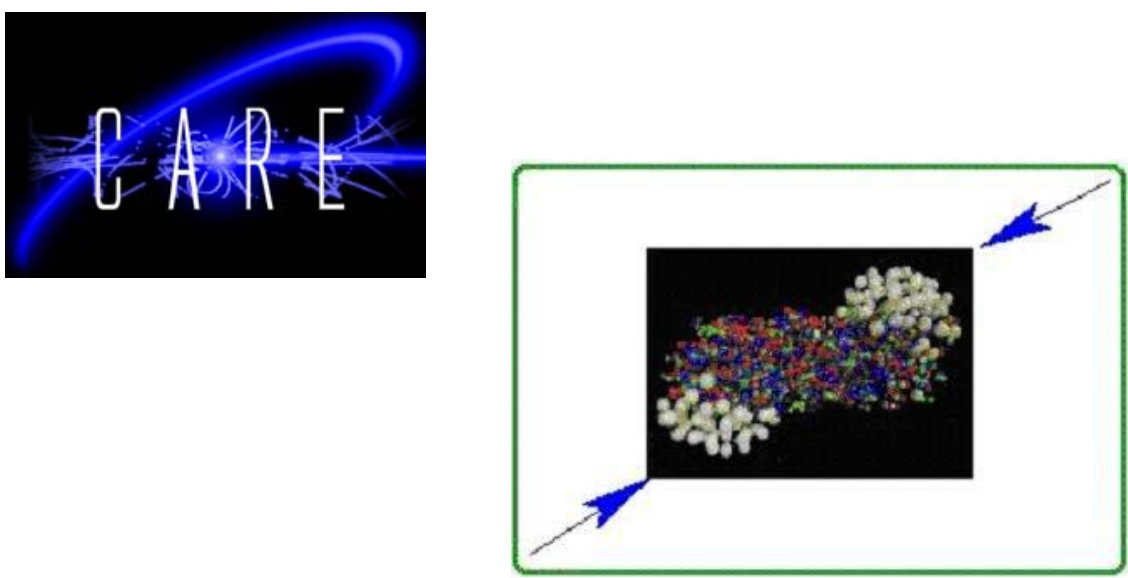

High Energy

High Intensity

Hadron Beams

\title{
A Concept for the LHC Luminosity Upgrade Based on Strong Beta* Reduction Combined with a Minimized Geometrical Luminosity Loss Factor
}

\author{
J.-P. Koutchouk ${ }^{1}$, R. Assmann ${ }^{1}$, E. Métral ${ }^{1}$, E. Todesco ${ }^{1}$, F. Zimmermann ${ }^{1}$, R. De Maria ${ }^{1,2}$, \\ G. Sterbini ${ }^{1,2}$ \\ 1) CERN, Geneva, Switzerland \\ 2) EPFL, Lausanne, Switzerland
}

\begin{abstract}
A significant reduction of $\beta^{*}$ requires a new beam crossing scheme to overcome the consequence of the luminosity loss factor. We define in this paper the parameters of a possible solution taking advantage of an early separation scheme optionally supplemented by a weak crab crossing. Large aperture $(150 \mathrm{~mm}) \mathrm{Nb}_{3} \mathrm{Sn}$ triplet quadrupoles are chosen for their compactness, temperature margin and ability to relax the collimator gap and impedance. This concept offers high performance for a moderate increase of complexity. An open issue is the possibility of integrating dipoles inside the detectors.
\end{abstract}

Contribution to the " $22^{\text {nd }}$ Particle Accelerator Conference PAC07", Albuquerque (USA), 25-29 June 2007

Work supported by the European Community-Research Infrastructure Activity under the FP6 "Structuring the European Research Area" programme (CARE, contract number RII3-CT2003-506395) 


\title{
A CONCEPT FOR THE LHC LUMINOSITY UPGRADE BASED ON STRONG BETA* REDUCTION COMBINED WITH A MINIMIZED GEOMETRICAL LUMINOSITY LOSS FACTOR
}

\author{
J-P. Koutchouk, R. Assmann, E. Métral, E. Todesco, F. Zimmermann, CERN, Geneva, Switzerland \\ R. De Maria, G. Sterbini, CERN, Geneva \& EPFL, Lausanne, Switzerland
}

\section{Abstract}

A significant reduction of $\beta^{*}$ requires a new beam crossing scheme to overcome the consequence of the luminosity loss factor. We define in this paper the parameters of a possible solution taking advantage of an early separation scheme optionally supplemented by a weak crab crossing. Large aperture $(150 \mathrm{~mm}) \mathrm{Nb}_{3} \mathrm{Sn}$ triplet quadrupoles are chosen for their compactness, temperature margin and ability to relax the collimator gap and impedance. This concept offers high performance for a moderate increase of complexity. An open issue is the possibility of integrating dipoles inside the detectors.

\section{MOTIVATION \& CONCEPT}

Increasing the beam intensity in the LHC is extremely challenging. We rather investigate here the potential of increasing the focusing of the beam at the crossing point (IP). It indeed only requires a localized modification of the machine with the potential of a fast and reliable increase of performance. To prevent parasitic beam interactions in the common sections, the crossing angle shall however increase with the focusing, cancelling the best part of the performance improvement. To overcome this difficulty, we consider the use of an early separation scheme [1] that may optionally be backed by a weak crab crossing scheme [2]. A long-range wire or electron lens compensation may further reduce the required residual crossing angle. It has been shown [3] that this class of solution offers a large luminosity increase. Two solutions are studied here for two distances $l^{*}$ of the triplet from the IP (23 and $13 \mathrm{~m}$ ) and the planned ultimate bunch charge.

\section{THE GEOMETRICAL LOSS FACTOR}

With the nominal bunch spacing of $25 \mathrm{~ns}$, there are about $k=33$ beam interactions per experimental point. A crossing angle $\theta_{c}$ separates the beams except at the IP; its value depends on the beta function at the IP $\beta^{*}$ and on the beam current according to the semi-empirical law [4]:

$$
\theta_{c}=\theta_{0} \sqrt{\beta_{0}^{*} / \beta^{*}}\left(\frac{6.5}{9.5}+\frac{3}{9.5} \sqrt{\frac{k N_{p}}{k_{0} N_{p 0}}}\right)
$$

where $N_{p}$ is the bunch charge. This angle causes a significant loss in luminosity, given by the luminosity loss factor:

$$
R_{g}^{c}=\frac{1}{\sqrt{\pi} \sigma_{z}} \int_{-\infty}^{\infty} d s\left(\exp \left[-\frac{s^{2}}{\sigma_{z}^{2}}-\frac{\theta_{c}^{2}\left(-k_{c} s+\sin \left(k_{c} s\right)\right)^{2}}{4 k_{c}^{2} \sigma_{x}^{* 2}\left(1+\frac{s^{2}}{\beta^{* 2}}\right)}\right] \frac{1}{\left(1+\frac{s^{2}}{\beta^{* 2}}\right)}\right)(2)
$$

This expression includes the effect of a finite crab radiofrequency wavelength and the hourglass effect [5], with $\sigma_{z}$ rms bunch length and $\kappa_{c}$ number of bunches. The dependency of the factor $R$ on $\beta^{*}$ (Fig. 1) shows the necessity to counteract the negative impact of the crossing angle as $\beta^{*}$ is reduced.

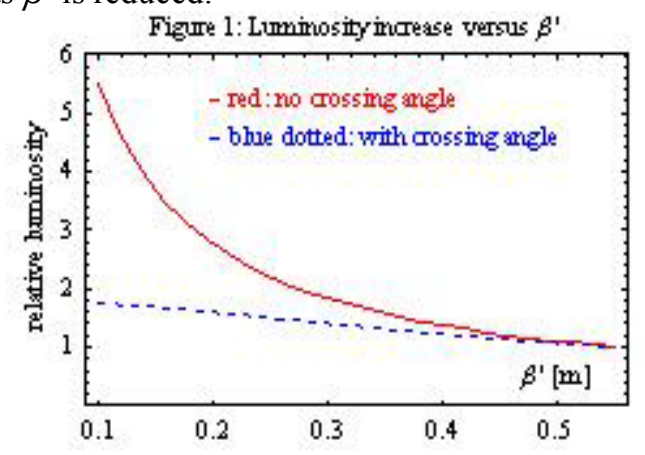

\section{EARLY SEPARATION SCHEME}

The ideal early separation scheme [1] would allow the beams to collide exactly head-on at the IP and to separate them before the first parasitic encounter about $3.5 \mathrm{~m}$ downstream, using a dipole "D0" placed inside the detector. A regular orbit corrector in front of the first quadrupole limits the beam separation to the required value. In practice, the first location available for a dipole is at $4 \mathrm{~m}$ from the IP; it is therefore necessary to leave a residual crossing angle sufficient to prevent a significant beam perturbation caused by one interaction on either side of each IP. Tracking studies [1] show that a residual crossing angle halved with respect to (1) appears to be tolerable. An experiment will be carried out in RHIC to validate this choice. Figure 2 shows the beam trajectory and normalized separation for $l^{*}=23 \mathrm{~m}$. The results for

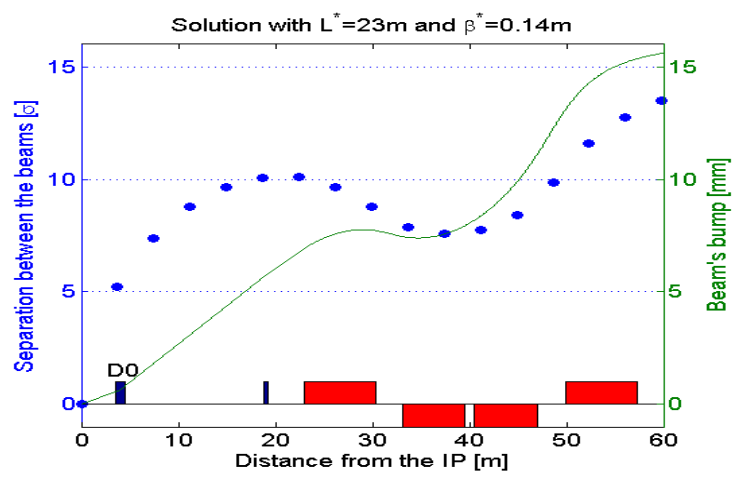

Figure 2: Beam trajectory and separation for $l^{*}=23 \mathrm{~m}$. 
$l^{*}=13 \mathrm{~m}$ is very similar. The parameters of the two solutions are given in table 1 . The D0 is assumed made of $\mathrm{Nb}_{3} \mathrm{Sn}$, with a length of $1 \mathrm{~m}$. The preliminary energy deposition studies and shielding of the D0 [6] show that the peak deposition can be kept below the operational limit of $\mathrm{Nb}-\mathrm{Ti}$ of $4 \mathrm{~mW} / \mathrm{cm}^{3}$. The albedo to the detector is potentially a serious issue that will now be studied. The early separation scheme can be used as well to level the luminosity in an operationally convenient way [7].

Table 1: Parameters of the D0 dipole for the two layouts

\begin{tabular}{|c|c|c|c|c|c|}
\hline $\begin{array}{c}l^{*} \\
{[\mathrm{~m}]}\end{array}$ & $\begin{array}{c}\beta^{*} \\
{[\mathrm{~cm}]}\end{array}$ & $\begin{array}{c}\theta_{c} \\
{[\mu \mathrm{rad}]}\end{array}$ & $\begin{array}{c}\text { D0 } \\
\text { strength } \\
{[\mathrm{T} \mathrm{m}]}\end{array}$ & $\begin{array}{c}\text { corrector } \\
\text { strength } \\
{[\mathrm{T} \mathrm{m}]}\end{array}$ & $\begin{array}{c}R_{g}^{c} \\
(\mathrm{w} / \mathrm{o} \mathrm{D} 0)\end{array}$ \\
\hline 23 & 14 & 302 & 4.46 & 0.94 & $0.59(0.35)$ \\
\hline 13 & 11 & 340 & 5.95 & 1.99 & $0.50(0.28)$ \\
\hline
\end{tabular}

\section{REQUIREMENTS FROM COLLIMATION}

(1) The IR triplets must be fully shadowed by the collimation system $[8,9]$. Their gaps are already tight and the triplet aperture should be increased.

(2) For the nominal LHC, it is expected that the beam will become unstable due to collimator-driven impedance for collimation settings as they are required for a $\beta^{*}$ of about $0.8 \mathrm{~m}$. Figure 3 shows the coherent tune shift for the most unstable coupled bunch mode due to the estimated machine impedance (including all the collimators) for various gaps and beam intensities. The two stability limits are obtained for maximum positive or negative anharmonicity by the Landau octupoles, which clearly has a modest effect. This analysis shows that the collimator gap shall be increased by $50 \%$ to $100 \%$ (6 to $12 \sigma)$ in a simplified scenario where all collimators are at the same $\beta$ function.

(3) Cleaning efficiency shall however not be compromised. Single-diffractive scattering is a limiting physical process. Increasing the collimator gaps requires the implementation of collimation phase 2 [9]. In

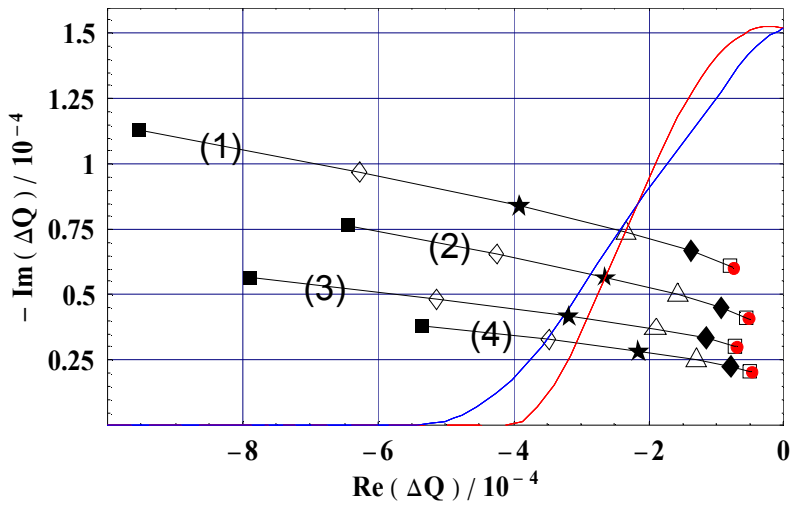

Figure 3: Stability limits: $25 \mathrm{~ns}$ spacing with ultimate bunch charge (1) and nominal bunch charge (2), $50 \mathrm{~ns}$ spacing with ultimate bunch charge (3), and nominal bunch charge (4). Nominal collimator gap (black squares), no collimators (red square), and intermediate situations where the collimator gap is increased by $20 \%$, $50 \%$, and a factor 2,3 and 10 .

05 Beam Dynamics and Electromagnetic Fields addition, the consequence of the larger chromatic beta beating requires evaluation.

(4) The tertiary collimators in the IR's may require hardware changes to maintain their functionality.

\section{LAYOUT AND OPTICS}

We consider a triplet of four quadrupole blocks, e.g. $\mathrm{F}[\mathrm{DD}] \mathrm{F}$, with two different lengths and identical or similar gradients. Using a parametric analysis we selected the solution that provides the smallest $\beta$ function in the IP, keeping the constraint of correcting the linear chromaticity with the available sextupoles. The quadrupole aperture is computed according to the guidelines described in [10], with 6 additional $\sigma$ (in diameter) to increase the collimator gap. Two cases are analysed: the nominal distance to the IP of $l^{*}=23 \mathrm{~m}$, and a reduced distance of $l^{*}=13 \mathrm{~m}$. (see Table 2). A $\beta^{*}$ of 0.11 $0.14 \mathrm{~m}$ can be reached with $\mathrm{Nb}_{3} \mathrm{Sn}$ quadrupoles of 150 $\mathrm{mm}$ aperture. For both layouts, an exactly optically matched solution has been found. The matching section and the dispersion suppressor are as in the nominal layout, with the exception of Q6, which is doubled.

Table 2: Lay-out parameters of two insertions

\begin{tabular}{ccccc}
\hline \hline distance to IP & $l^{*}$ & $(\mathrm{~m})$ & 23 & 13 \\
quad length & $l(Q 1-Q 3)$ & $(\mathrm{m})$ & 7.3 & 9.3 \\
quad length & $l(Q 2)$ & $(\mathrm{m})$ & 6.5 & 6.8 \\
total quad length & $l_{q}$ & $(\mathrm{~m})$ & 27.5 & 32.1 \\
triplet length & $l_{t}$ & $(\mathrm{~m})$ & 34.1 & 38.7 \\
Gradient Q1 & $G_{1}$ & $(\mathrm{~T} / \mathrm{m})$ & 166 & 169 \\
Gradient Q2 & $G_{2}$ & $(\mathrm{~T} / \mathrm{m})$ & 166 & 169 \\
Gradient Q3 & $G_{3}$ & $(\mathrm{~T} / \mathrm{m})$ & 166 & 141 \\
B peak estimate & $B_{p}$ & $(\mathrm{~T})$ & 13.7 & 13.5 \\
Aperture & $\phi$ & $(\mathrm{m})$ & 0.150 & 0.145 \\
$\beta$ funct. in IP & $\beta^{*}$ & $(\mathrm{~m})$ & 0.142 & 0.112 \\
Max $\beta$ funct. in Q1-Q3 & $\beta_{\max }$ & $(\mathrm{m})$ & 18700 & 16800 \\
\hline \hline
\end{tabular}

\section{CHROMATICITY AND ABERRATIONS}

The linear chromaticity correction is carried out with the lattice sextupoles, the defocusing ones being powered at $100 \%$ and the focusing ones at $60 \%$. The second order term is minimized through a phasing of the two IP's and the residual part is corrected by the individual powering of the arc sextupole families [11].This also corrects the off-momentum beta-beating in the triplet and in half of the machine [12]. In the other half of the machine it reaches $50 \%$ at $\Delta p / p=3 \times 10^{-4}$.

The dynamic aperture at collision is dominated by the field errors in the triplet. We assume that the field harmonics of the IR quadrupoles belong to a centred Gaussian distribution, i.e. that the allowed harmonics have been set to zero through a careful design. The random components are evaluated by scaling the existing triplet field quality [13]. Results show that the dynamic aperture is larger than $14 \sigma$, i.e. even larger than that of the nominal LHC.

D01 Beam Optics - Lattices, Correction Schemes, Transport 


\section{CRAB CROSSING OPTION}

The residual crossing angle at the IP is of the order of 0.3 mrad (Table 1). It causes a luminosity loss of $40 \%$ to $50 \%$ that could be avoided with a weak crab crossing device (Eq. (2)). Although global crab cavities appear applicable for angles lower than $1 \mathrm{mrad}$ [2], the global solution induces constraints and possible side effects. We choose here the more conservative local crab crossing studied in [5]. For a numerical estimate, the following assumptions

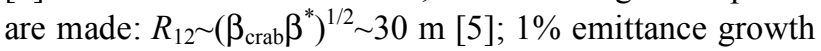
per hour, with an IP beam size of $7.4 \mu \mathrm{m}$, and a feedback gain of 0.2 , following the analysis in [14].

Table 3: Crab cavity voltage and noise tolerance for 0.3 mrad versus crab rf frequencies

\begin{tabular}{|l||c|c|}
\hline & Voltage & tolerance \\
\hline $800 \mathrm{MHz}$ & $2.1 \mathrm{MV}$ & $0.07^{\circ}, 80 \mathrm{fs}$ \\
\hline \hline $400 \mathrm{MHz}$ & $4.2 \mathrm{MV}$ & $0.04^{\circ}, 80 \mathrm{fs}$ \\
\hline
\end{tabular}

For comparison, the crab cavities recently installed at KEKB provide about $1.5 \mathrm{MV}$ crab voltage at $500 \mathrm{MHz}$ and the European X-Ray FEL requires a phase tolerance of $20 \mathrm{fs}$. The phase tolerance could be further relaxed with a flat beam design in the horizontal plane.

\section{PERFORMANCE PROSPECTS}

The estimates of the peak luminosity are given in Table 4. Each option (early separation, crab crossing) roughly doubles the peak luminosity. The performance loss resulting from $50 \mathrm{~ns}$ bunch spacing is compensated by the early separation scheme reaching full efficiency. Moving the triplet as close as possible from the IP is only significant for collinear head-on collisions $(+20 \%)$.

Table 4: Peak luminosity improvement versus options for nominal (slanted) and ultimate bunch charge

\begin{tabular}{|l|c|l|l|c|c|}
\hline$\#$ & \multirow{2}{*}{$\begin{array}{l}\text { Spacing } \\
{[\mathrm{ns}]}\end{array}$} & \multirow{2}{*}{ D0 } & Crab & \multicolumn{2}{|c|}{$\mathrm{L} / \mathrm{L} 0$} \\
\cline { 5 - 6 } & & & $1^{*}=23 \mathrm{~m}$ & $1^{*}=13 \mathrm{~m}$ \\
\hline 1 & 25 & No & No & 1.5 & 1.4 \\
\cline { 5 - 6 } & & & & 3.0 & 3.0 \\
\hline 2 & 25 & Yes & No & 2.5 & 2.6 \\
\cline { 5 - 6 } & & & & 5.2 & 5.3 \\
\hline 3 & 25 & Yes & Yes & 4.2 & 5.0 \\
\cline { 5 - 6 } & & & & 9.1 & 11.0 \\
\hline 4 & 50 & Yes & - & 2.1 & 2.5 \\
\cline { 4 - 6 } & & & & 4.6 & 5.5 \\
\hline
\end{tabular}

The fast decay rate of the luminosity is not mitigated by an increased beam current. Following [15], starting from 10 (or 5) $\times 10^{34} \mathrm{~cm}^{-2} \mathrm{~s}^{-1}$, the luminosity is reduced by a factor of 2 in 3.5 (or 7) hours. For optimal run duration of 6.5 (or 9) hours, a turn-around time of 5 hours, the average luminosity is typically $40 \%$ of the peak luminosity.

To avoid this fast luminosity decay, an operationally simple luminosity levelling is obtained by adjusting the crossing angle with the early separation scheme [5], preventing the side effects that can plague other methods.
Simulations have shown the capability e.g. of a constant luminosity for 4 hours with a marginal cost in integrated luminosity (10 to $15 \%)$.

A luminosity increase by a factor of 10 will require a learning period. Experience with other colliders [16] shows that, in an optimistic scenario of moderate complexity, 4 years are necessary to multiply by 10 the luminosity by a beam current increase. In comparison, only a few weeks were sufficient to reduce by a factor 7.2 the $\beta^{*}$ when the first superconducting low-beta insertion was installed in the ISR. A conservative 6 months to one year to reach in LHC the corresponding luminosity increase seems realistic. For a typical running period of 5 years, this faster rise of performance after a well mastered optics change doubles the effective integrated luminosity.

\section{CONCLUSIONS}

This study shows that, provided an early separation scheme can be implemented inside the detectors, a significant increase of luminosity may be reached with a minimized increase of machine complexity. The fast decay of luminosity can be compensated by an operationally simple luminosity levelling scheme. The rate of rise of performance should be fast, due to local modifications only and a comfortable quadrupole aperture. The risks are mitigated by the possibility of using two independent crossing schemes that can be supplemented by wire and electron lens compensation.

We acknowledge the support of the European Community-Research Infrastructure Activity under FP6 "Structuring the European Research Area" program (CARE, contract number RII3-CT-2003-506395).

\section{REFERENCES}

[1] J.-P. Koutchouk, G. Sterbini, WEPCH094, EPAC06.

[2] R. Calaga, et al, Proceedings LUMIO6, Valencia.

[3] J.-P. Koutchouk, MOPLS011, EPAC06.

[4] Y. Papaphilippou, F. Zimmermann, CERN-SL-99-039 $A P(1999) 103$.

[5] F. Zimmermann, U. Dorda, LUMI05, CERN-2006008 p. 55.

[6] G. Sterbini, et al, "Layout V1 for the early separation scheme in ATLAS", to be published.

[7] G. Sterbini, J.-P Koutchouk, LHC Pr. Note 403(2007).

[8] R. Assmann et al, TUODFI01, EPAC06 \& CERN LHC Pr. Report 919.

[9] R. Assmann, Chamonix XII p. 163 (2003).

[10] E. Todesco, J.-P. Koutchouk, LUMI06, Valencia.

[11] R. De Maria, ICAP06 p. 213 (2006).

[12] S. Fartoukh, LHC Project Report 308 (2001).

[13] B. Bellesia, et al., Phys. Rev. STAB 10 (2007) 062401.

[14] Y.I. Alexahin, NIM A 391 (1996) 73.

[15] F. Zimmermann, these proceedings.

[16] V. Shiltsev, FNAL TD-1101 (2006).

D01 Beam Optics - Lattices, Correction Schemes, Transport 